\title{
Minimally Invasive Versus Conventional Aortic Valve Replacement
}

\section{A Propensity-Matched Study From the UK National Data}

\author{
Rizwan Q. Attia, MRCS, * Graeme L. Hickey, PhD, $\neq$ Stuart W. Grant, MRCS, $\$$ \\ Ben Bridgewater, FRCS, $\dagger+\S$ James C. Roxburgh, FRCS, * Pankaj Kumar, FRCS,// \\ Paul Ridley, FRCS,I Moninder Bhabra, FRCS,\# Russell W. J. Millner, FRCS, ** \\ Thanos Athanasiou, FRCS, $\dagger+$ Roberto Casula, FRCS, $\dagger \dagger$ Andrew Chukwuemka, FRCS, $\dagger \dagger$ \\ Thasee Pillay, FRCS, $\neq$ * and Christopher P. Young, FRCS*
}

\begin{abstract}
Objective: Minimally invasive aortic valve replacement (MIAVR) has been demonstrated as a safe and effective option but remains underused. We aimed to evaluate outcomes of isolated MIAVR compared with conventional aortic valve replacement (CAVR).

Methods: Data from The National Institute for Cardiovascular Outcomes Research (NICOR) were analyzed at seven volunteer centers (2006-2012). Primary outcomes were in-hospital mortality and midterm survival. Secondary outcomes were postoperative length of
\end{abstract}

Accepted for publication October 28, 2015.

From the *Department of Cardiothoracic Surgery, Guy's and St Thomas' Hospital, London, UK; †Centre for Health Informatics, Manchester Academic Health Science Centre, University of Manchester, Manchester, UK; \$National Institute for Cardiovascular Outcomes Research, Institute of Cardiovascular Science, University College London, London, UK; §Department of Cardiothoracic Surgery, Manchester Academic Health Science Centre, University Hospital of South Manchester, Wythenshawe, UK; |Department of Cardiothoracic Surgery, Morriston Hospital, Morriston, Swansea, UK; @Department of Cardiothoracic Surgery North Staffordshire Royal Infirmary, University Hospital of North Staffordshire NHS Trust, Stoke-on-Trent, UK; \#Department of Cardiothoracic Surgery, Heart and Lung Centre, New Cross Hospital, Wolverhampton, UK; **Department of Cardiothoracic Surgery, Lancashire Cardiac Centre, Victoria Hospital NHS Trust, Blackpool, UK; $\uparrow$ † Department of Cardiothoracic Surgery, Hammersmith Hospital, London, UK; and \$\$Department of Cardiothoracic Surgery, The Cardiothoracic Centre, Freeman Hospital, Newcastle upon Tyne, UK.

Presented at the Annual Scientific Meeting of the International Society for Minimally Invasive Cardiothoracic Surgery, June 3-6, 2015, in Berlin, Germany.

Supplemental digital content is available for this article. Direct URL citation appears in the printed text and is provided in the HTML and PDF versions of this article on the journal's website (www.innovjournal.com).

Disclosure: The authors declare no conflicts of interest.

Address correspondence and reprint requests to Rizwan Q. Attia, MRCS, Department of Cardiothoracic Surgery, St Thomas' Hospital, 6th Floor East Wing, Westminster Bridge Rd, London, SE1 7EH, UK. E-mail: rizwanattia@, doctors.org.uk.

Copyright (c) 2016 by the International Society for Minimally Invasive Cardiothoracic Surgery

This is an open-access article distributed under the terms of the Creative Commons Attribution-Non Commercial-No Derivatives License 4.0 (CCBY-NC-ND), where it is permissible to download and share the work provided it is properly cited. The work cannot be changed in any way or used commercially.

ISSN: 1556-9845/16/1101-0015 stay as well as cumulative bypass and cross-clamp times. Propensity modeling with matched cohort analysis was used.

Results: Of 307 consecutive MIAVR patients, 151 (49\%) were performed during the last 2 years of study with a continued increase in numbers. The 307 MIAVR patients were matched on a 1:1 ratio. In the matched CAVR group, there was no statistically significant difference in in-hospital mortality [MIAVR, 4/307,(1.3\%); $95 \%$ confidence interval (CI), 0.4\%-3.4\% vs CAVR, $6 / 307$ (2.0\%); $95 \%$ CI, $0.8 \%-4.3 \%$; $P=0.752]$. One-year survival rates in the MIAVR and CAVR groups were $94.4 \%$ and $94.6 \%$, respectively. There was no statistically significant difference in midterm survival $(P=0.677$; hazard ratio, 0.90 ; $95 \% \mathrm{CI}, 0.56-1.46)$. Median postoperative length of stay was lower in the MIAVR patients by 1 day $(P=0.009)$. The mean cumulative bypass time ( 94.8 vs 91.3 minutes; $P=0.333$ ) and cross-clamp time (74.6 vs 68.4 minutes; $P=0.006$ ) were longer in the MIAVR group; however, this was significant only in the cross-clamp time comparison. Conclusions: Minimally invasive aortic valve replacement is a safe alternative to CAVR with respect to operative and 1-year mortality and is associated with a shorter postoperative stay. Further studies are required in high-risk (logistic EuroSCORE $>10$ ) patients to define the role of MIAVR.

Key Words: Aortic valve disease, Aortic valve replacement, Minimally invasive surgery.

(Innovations 2016;11:15-23)

nterest in minimally invasive aortic valve replacement (MIAVR) has increased after the adoption of transcatheter techniques to treat aortic stenosis and early feasibility studies on sutureless valve techniques. ${ }^{1-6}$ Numerous minimally invasive surgical approaches for aortic valve replacement (AVR) have been proposed including upper or lower hemisternotomy, right parasternal minithoracotomy, and transverse sternotomy. ${ }^{7-9}$ Many reports including prospective randomized studies and meta-analyses have demonstrated advantages of these incisions, which include reduced pain, reduced surgical trauma, less bleeding, earlier functional recovery, reduced incidence of chest and sternal wound infections, lower incidence of arrhythmias, shorter hospital stay, improved cosmetics, and reduced costs. ${ }^{10-15}$ Despite these 
potential advantages, there has been limited uptake of these techniques with only $5 \%$ to $10 \%$ of isolated AVRs being performed in the USA and across Europe. ${ }^{12,16,17}$ The potential drawbacks of minimally invasive cardiac surgery are perceived increases in operative and cross-clamp times that may place patients at increased risk of adverse outcomes.

There are no clear data guiding clinical practice with regard to which patient groups would benefit from minimally invasive approaches. The objective of this research was to investigate the current real-world experience with MIAVR and report contemporaneous multicenter results for MIAVR versus a propensity-matched cohort of conventional AVRs (CAVRs) within the same institutions.

\section{METHODS}

\section{Data Source, Validation, and Study Population}

Prospectively collected data were extracted from The National Institute for Cardiovascular Outcomes Research, National Adult Cardiac Surgery Audit (NACSA) database in November 2013 for all adult cardiac surgery procedures performed in the UK. As described elsewhere, reproducible cleaning algorithms were applied to the database to clean the data. ${ }^{18}$ Briefly, duplicates were removed; transcriptional discrepancies were harmonized; conflicting data and extreme values were corrected or removed. The data are returned annually to each unit for local validation as part of the NACSA program.

For this study, procedures were included if they corresponded to an isolated AVR (conventional or minimally invasive) performed in a participating hospital with a procedure date between December 1, 2006, and November 30, 2012. Transcatheter aortic valve implantations (TAVIs) were identified and excluded. Patients were identified as having undergone MIAVR using pseudoanonymized National Health Service (NHS) or hospital numbers supplied by each participating center; all other patients were assumed to have undergone CAVR. Exclusion criteria for this study were indication (or missing data) of nonelective surgery, previous cardiac surgery, critical preoperative state, use of preoperative intravenous nitrates, and renal dysfunction (defined as patient on dialysis or preoperative serum creatinine $>200 \mu \mathrm{mol} / \mathrm{L}$ ).

Follow-up data up until the point of discharge were collected through the NACSA, with postdischarge survival data collected through linkage to the Office for National Statistics registry. For a small number of patients discharged alive with no available record linkage, the patients were followed up to the point of discharge only. Database variable definitions used for the study are available at http:/www.ucl.ac.uk/nicor/ audits/Adultcardiacsurgery/datasets, and variables generated within this study predominately follow the definitions as given by the EuroSCORE model. ${ }^{19}$

\section{Outcome Measures}

The primary outcomes for this study were (1) in-hospital mortality defined as death due to any cause during admission to the base hospital for cardiac surgery and (2) midterm survival. Secondary outcomes were postoperative length of stay (PLOS), cumulative bypass time, and cumulative cross-clamp times. Patients who died in the hospital on the day of surgery were recorded as having a PLOS of 0.5 days.

\section{Missing Data}

All missing patient characteristic and preoperative data were imputed using a chain equation method that generates "plausible" synthetic values for each missing risk factor. Because the amount of missing data were low, a single imputation approach was preferred to multiple imputations. Variables used in the imputation models included those listed in Table 1, a hospital-level indicator and in-hospital mortality. ${ }^{20}$ Imputation was implemented using the MICE package (version 2.18) in $\mathrm{R} .{ }^{21}$ All analyses from here onward are based on the imputed data. Missing outcome data (bypass time, cross-clamp time, PLOS) were evaluated on a case-complete basis.

\section{Propensity Score Matching}

A propensity-matched analysis was used to account for inherent confounding. ${ }^{22}$ The propensity score model was developed by fitting a nonparsimonious multiple logistic regression models with AVR type (minimally invasive vs conventional) as the outcome variable with independent variables as listed in Table 1. This model was used to calculate a propensity score for each record. Patients who received MIAVR were then matched (1-to-1 matching) to patients who underwent CAVR using nearest neighbor matching with a greedy matching algorithm.

After matching, the balance between the two groups was evaluated by comparing the patient and preoperative characteristics using standardized differences and graphical comparison of propensity score distributions. An absolute standardized difference of less than $10 \%$ supports the assumption of balance between the two groups. ${ }^{23}$ Propensity matching and diagnostic checking were performed using the MatchIt package in $\mathrm{R}$ (version 2.4-21). ${ }^{22}$

\section{Statistical Analysis}

In the matched groups, in-hospital mortality was compared between the MIAVR and CAVR groups using Fisher exact test. In addition, an odds ratio was estimated with a corresponding $95 \%$ confidence interval (CI). Midterm survival was compared between the matched groups using KaplanMeier survival curve methodology stratified on operation type, and the log-rank test was used to test for differences between them. The hazard ratio for MIAVR was also estimated by fitting a Cox proportional hazards regression model with operation as the sole independent variable. Postoperative length of stay was compared between the two groups using a two-sided nonparametric Mann-Whitney $U$ test. Cumulative cross-clamp times and cardiopulmonary bypass (CPB) times were compared using an independent samples $t$ test.

For the analysis of outcomes in propensity score-matched groups, there is no general statistical consensus on whether an independent or matched-pairs analysis should be applied. ${ }^{24,25}$ We primarily focus on the independent groups analysis; however, as sensitivity analysis, the data have also been analyzed using methodology suitable for a matched-pairs design. Namely, for in-hospital mortality, McNemar test is used; for midterm survival-stratified log rank tests, stratifying on the matched-pairs is used; for PLOS, the Wilcoxon signed rank test is used; and for cross-clamp and CPB time comparisons, a paired $t$ test is applied. All analyses were 
TABLE 1. Patient Demographics and Risk Factors in the Unmatched and Propensity Matched MIAVR and CAVR Groups

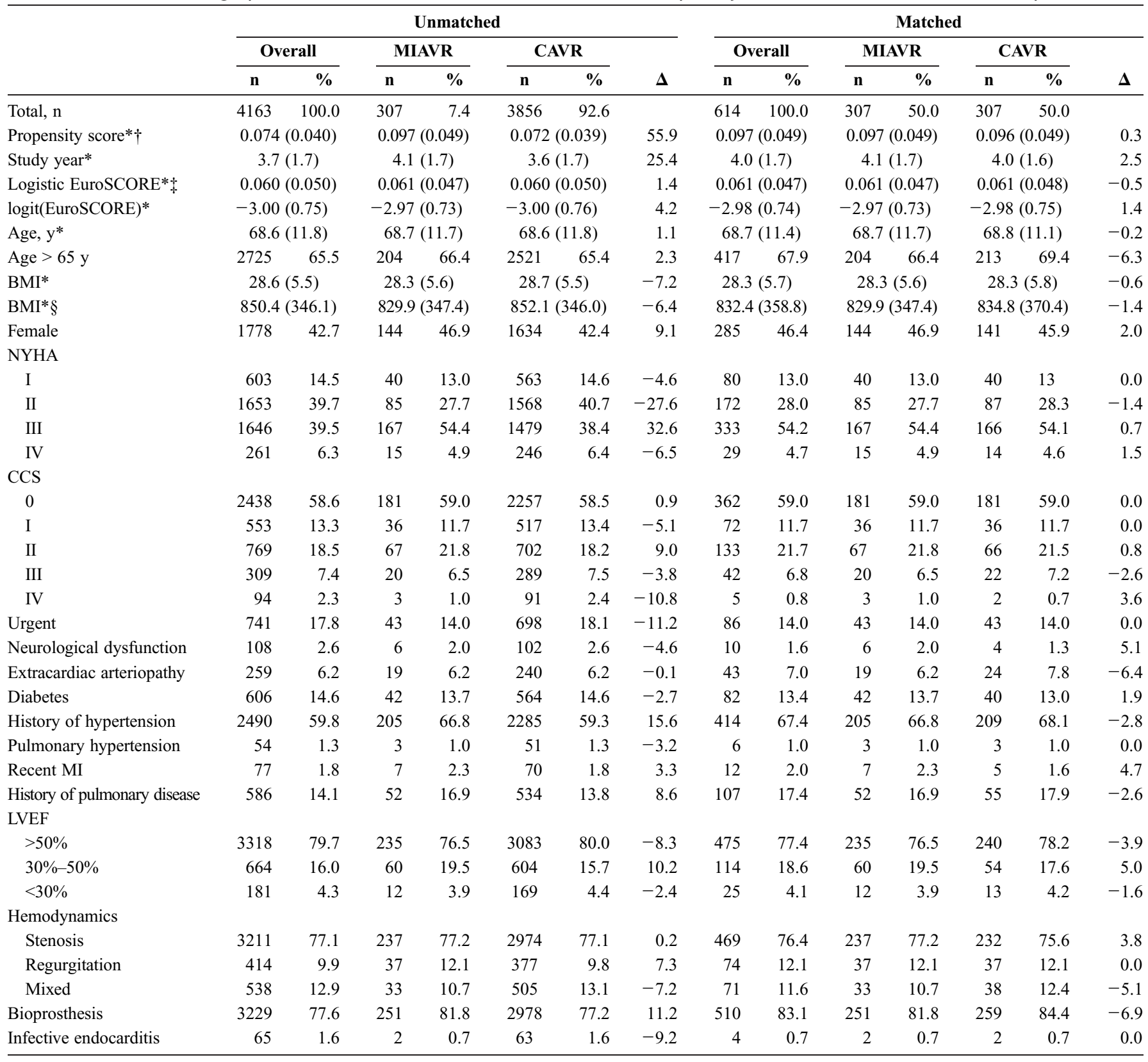

*Continuous variables reported as mean (SD).

$\dagger$ Calculated from the propensity score model. Not an independent variable itself.

†Logistic EuroSCORE was not included in the propensity score. Instead logit-transformed EuroSCORE was included

$\S$ Number of patients.

Standardized difference $(\Delta)$ is used to measure the degree of balance between the MIAVR and CAVR cohorts before and after matching.

BMI, body mass index; CAVR, conventional aortic valve replacement; CCS, Canadian Cardiac Society; MI, myocardial infarction; MIAVR, minimally invasive aortic valve replacement; NYHA, New York Heart Association.

performed in R version 3.0.2 (R Development Core Team, Vienna, Austria). ${ }^{26}$ In all cases, a $P<0.05$ was considered significant.

\section{Operative Technique}

The patients were operated on according to standard AVR techniques. We report the St Thomas' technique that is typical of the minimally invasive strategy. The contributing units have minor variations to this technique and differences dictated by patient-specific anatomical and physiological factors.

The patients were anesthetized in the supine position and intubated with a single-lumen endotracheal tube. Defibrillator pads were placed over the chest wall and back. A transvenous 


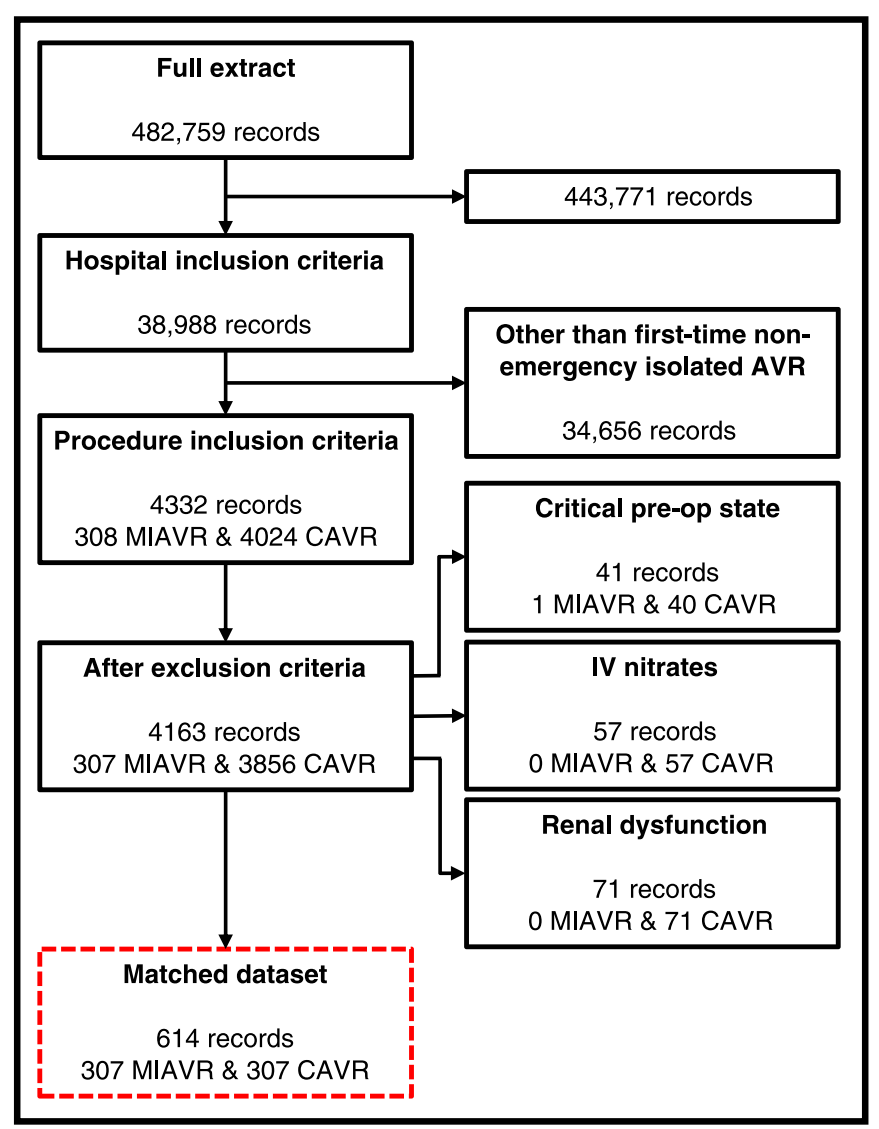

FIGURE 1. Flow diagram demonstrating patient selection, inclusion, and exclusions.

pacing system was inserted at some centers via the internal jugular vein. Transesophageal echocardiography was used routinely in most cases to allow assessment of aortic valve anatomy, annular sizing, deairing, and assessment of postoperative valve and cardiac function. After skin preparation and draping, a 4- to 6-cm skin incision usually an upper $\mathrm{J}$ hemisterntomy through the third or fourth intercostal space was performed. To enable smaller venous cannulae, vacuumassisted drainage was used in some patients. A triple-stage venous cannula was used in most cases, placed in the superior vena cava. Some patients necessitated peripheral cannulation, depending on body habitus and cardiac anatomy. The aorta was cannulated for arterial return. The majority of cases used mild hypothermic $32^{\circ} \mathrm{C}$ to normothermic CPB with aortic crossclamping. Antegrade blood cardioplegia was used in most cases. Aortic valve replacement was performed using interrupted or semicontinuous technique as per surgeon preference. Before closure of the aorta, the operative field was flooded with $\mathrm{CO}_{2}$ to minimize the chance of air embolism. The lungs were also inflated to expel air from the left ventricle and aorta. Deairing was monitored using transesophageal echocardiography. On completion, the patient was decannulated, and ventricular pacing wire was placed. The sternum was closed with wires behind a standard chest or Blake drain. The wound was closed in layers.

\section{RESULTS}

\section{Data Set and Demographics}

After applying the study inclusion and exclusion criteria, a final cohort of 4163 procedures was identified (307 MIAVR and 3856 CAVR). Full details are provided in the data flow diagram in Figure 1. The proportion of missing data was less than $2 \%$ for all patient and operative characteristics, with the exception of neurological dysfunction $(5.0 \%)$ and pulmonary hypertension $(2.7 \%)$. Outcomes were complete as follows: inhospital mortality for all records $(100 \%)$, postdischarge discharge follow-up survival data in 4088 records (98.2\%), PLOS in 4123 records $(99.0 \%)$, as well as cumulative bypass and cross-clamp times present in $4098(98.4 \%)$ and $4103(98.6 \%)$ records, respectively.

The proportion of AVRs performed as MIAVR cases at the participating hospitals doubled during the 6-year study period from $5.3 \%$ to $10.0 \%$ (Fig. 2). Of the seven units included, there was one outlying center that performed $25.3 \%$ of AVR cases as MIAVR, whereas the others performed between $1.5 \%$ and $9.3 \%$ of cases as MIAVR (Fig. 3). The distributions of logistic EuroSCORE in the MIAVR and CAVR cohorts have remained comparable throughout the study (Supplementary Digital Content, http://links.lww.com/INNOV/A71).

\section{Propensity Score Matching}

All 307 MIAVR records were matched to 307 CAVR records. A summary of patient demographics and risk factors before and after propensity matching based on imputed data is given in Table 1. Before matching, there was imbalance in study year, with a $25.4 \%$ standardized difference in favor of

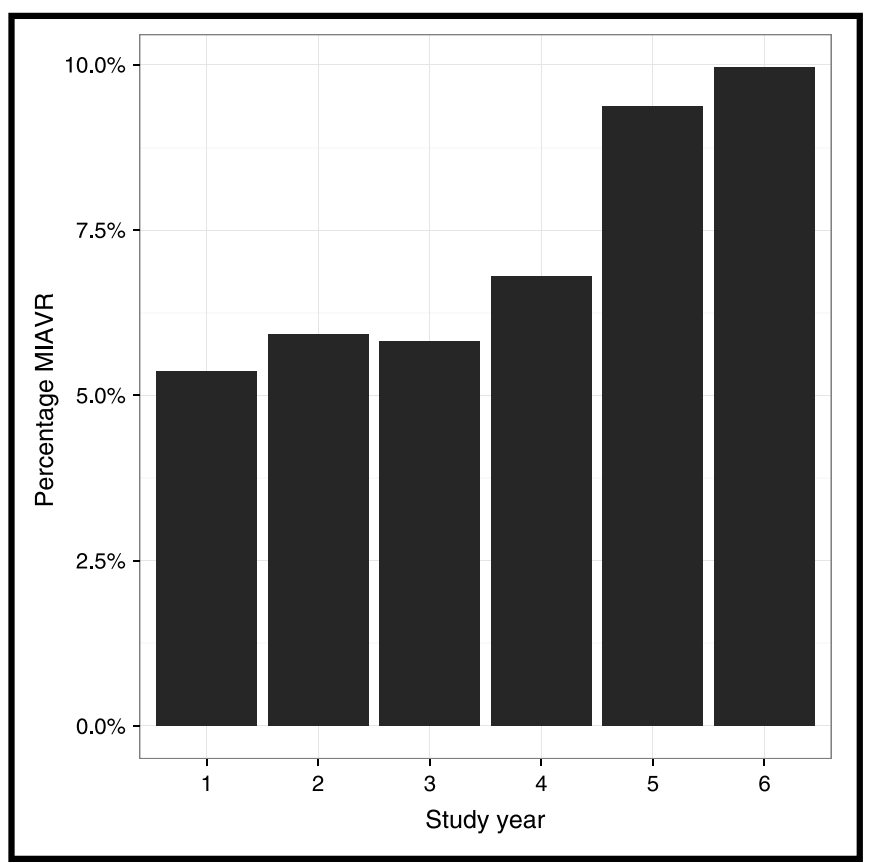

FIGURE 2. The yearly percentage of AVRs performed as MIAVR across all centers during the study period. AVR, aortic valve replacement; MIAVR, minimally invasive aortic valve replacement. 


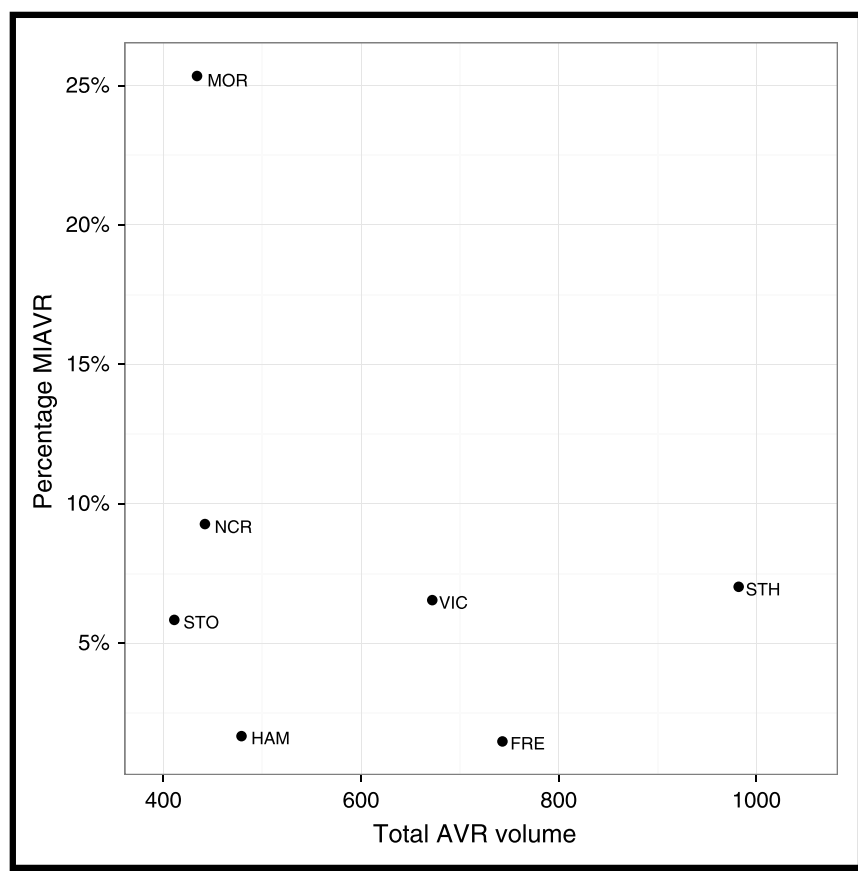

FIGURE 3. Volume of cases and rate of MIAVR across the participating units. FRE, Freeman Hospital; HAM, Hammersmith Hospital; MIAVR, minimally invasive aortic valve replacement; MOR, Morriston Hospital; NCR, New Cross Hospital; STH, St Thomas Hospital; STO, University Hospital of North Staffordshire; VIC, Victoria Hospital.

MIAVR, as an increasing number of MIAVR cases being performed in the latter half of the study; proportion of patients in New York Heart Association class IV (6.5), Canadian Cardiac Society IV (10.8), clinically urgent (11.2), being treated for infective endocarditis (9.2), or with neurological symptoms (4.6) was higher in the CAVR group. However, the absolute standardized differences were small $(<7 \%)$ for all variables after matching, and so the two cohorts were considered adequately balanced (Table 1). The distributions of propensity scores before and after matching are more closely aligned after matching.

\section{In-Hospital Mortality Analysis}

For the matched groups, there was no statistically significant difference in in-hospital mortality between the two groups $(P=0.663)$ : CAVR, 6/307 (2.0\%; 95\% CI: 0.8\%-4.3\%) versus MIAVR, $4 / 307$ (1.3\%; 95\% CI, 0.4\%-3.4\%), yielding an odds ratio of $0.66(95 \% \mathrm{CI}, 0.19-2.37)$ for MIAVR. Inferences were unchanged using matched-pairs analysis $(P=0.752)$.

\section{Survival Analysis}

Five records in the CAVR propensity-matched group could not be linked to the Office for National Statistics death registry. These records were therefore censored at point of discharge. Sensitivity analyses with the exclusions did not result in any changes in inferences. The median follow-up time was 2.6 years (interval range, 2 days to 6.7 years). There was no significant difference in midterm survival between the matched MIAVR and CAVR groups as shown in Figure $4(P=0.677)$.
The estimated hazard ratio in the matched groups was 0.90 (95\% CI, 0.56-1.46). Survival at 1 year in the MIAVR group was $94.4 \%(95 \% \mathrm{CI}, 91.9 \%-97.0 \%)$ and in the CAVR group was $94.6 \%(95 \%$ CI, 92.1\%-97.2\%). Survival at 5 years in the MIAVR group was $87.5 \%(95 \% \mathrm{CI}, 83.1 \%-92.2 \%)$ and in the CAVR group was $85.5 \%$ (95\% CI, 80.4\%-91.0\%). Inferences were unchanged using matched-pairs analysis $(P=0.668)$.

\section{Secondary Outcomes}

Postoperative length of stay was significantly longer in the CAVR matched group, with a difference in median values of 1 day $(P=0.004)$ (Table 2$)$. Inferences were unchanged using matched-pairs analysis $(P=0.009)$. The mean crossclamp and CPB times were 6.3 and 3.5 minutes longer for the MIAVR group, respectively (Table 2, Fig. 5); however, this difference was only significant for the cross-clamp times ( $P=0.006$ and $P=0.333$, respectively). Inferences were unchanged using matched-pairs analysis $(P=0.010$ and $P=0.355$, respectively).

\section{DISCUSSION}

This study demonstrates that MIAVR has comparable outcomes with those of CAVR with regard to in-hospital mortality and midterm survival when analyzed at 1 and 5 years. Small increases in cross-clamp and CPB times for MIAVR were demonstrated, but these are unlikely to have impact on outcomes. A statistically significant shorter PLOS was present in the MIAVR group. The strength of this study is the propensitymatched comparative analysis of MIAVR outcomes. This study combines contemporary national MIAVR experience to CAVR experience at the same cardiac centers and thus provides accurate real-world outcome data for MIAVR.

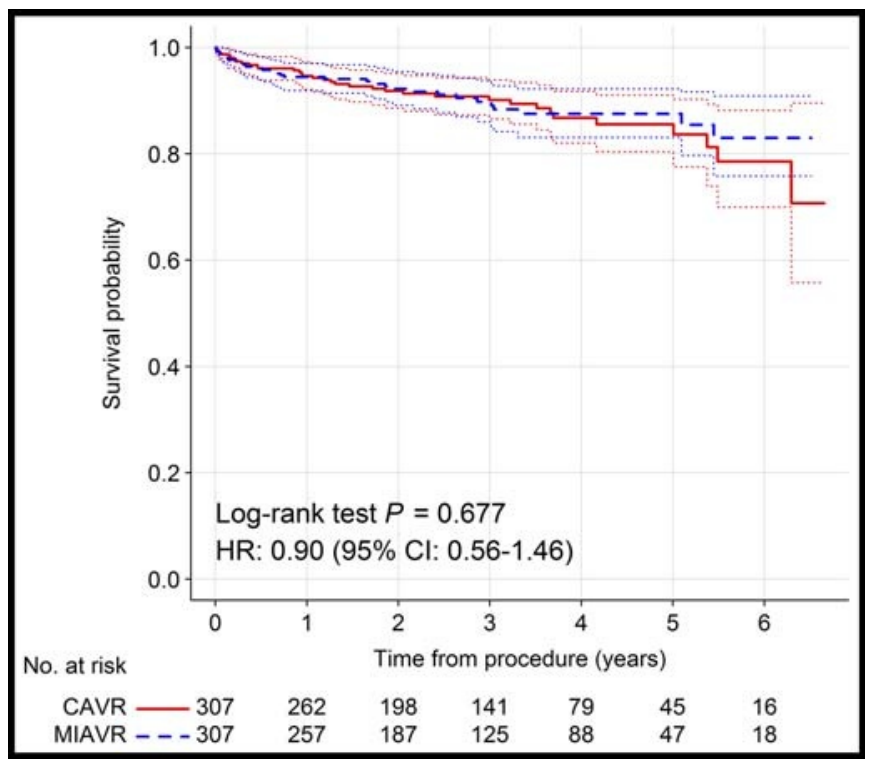

FIGURE 4. Kaplan-Meier survival curves for the propensity matched MIAVR and CAVR cohorts. Dashed lines indicate approximate $95 \%$ confidence intervals. CAVR, conventional aortic valve replacement; MIAVR, minimally invasive aortic valve replacement. 
TABLE 2. The Cardiopulmonary Bypass and Cross-Clamp Times and Postoperative Length of Stay Across the Propensity-Matched MIAVR and CAVR Cohorts

\begin{tabular}{lrrrr}
\hline & & & \multicolumn{1}{c}{} \\
\cline { 4 - 5 } & MIAVR & CAVR & Independent Samples Analysis & Matched-Pairs Analysis \\
\hline PLOS, ${ }^{*}$ median $(\mathrm{Q} 1-\mathrm{Q} 3), \mathrm{d}$ & $6(5-8)$ & $7(5-10)$ & 0.004 & 0.009 \\
CPB time, $\dagger$ mean (SD), min & $94.8(34.6)$ & $91.3(53.4)$ & 0.333 & 0.355 \\
Cross-clamp time, + mean (SD), min & $74.6(28.8)$ & $68.4(27.4)$ & 0.006 & 0.010 \\
\hline
\end{tabular}

*Two values were missing in the CAVR group.

$\dagger$ Five values were missing in the CAVR group and three values in the MIAVR group.

†Five values were missing in the CAVR group and two values in the MIAVR group.

CAVR, conventional aortic valve replacement; MIAVR, minimally invasive aortic valve replacement; PLOS, postoperative length of stay; Q1, first quartile; Q3, third quartile.

We wrote to all centers in the UK to invite them to participate in this study. Nine enthusiastic centers responded, and seven are included where the data collected were robust and complete; other units did not participate, and therefore, this work represents a snapshot of contemporary practice in the UK. The data demonstrates that MIAVRs are increasingly performed, with an increase in numbers as a total percentage of AVRs. The proportion doubles during the study period.

Based on the results of this study, MIAVR is a safe alternative to CAVR, with $98.6 \%$ of patients surviving to hospital discharge. The reduction in PLOS in the MIAVR group may be a surrogate marker for reduced postoperative complications. Survival at 1 year exceeds $94 \%$ for both MIAVR and CAVR, and there is no difference in midterm survival.

Although the percentage of MIAVRs increased during the study period, MIAVR still represents a small proportion of total caseload even at the enthusiastic centers participating in this study. There are wide variations in the number of centers undertaking MIAVR and the number of surgeons involved at those centers. Practice is known to range between surgeons who have a default minimally invasive approach where all first-time isolated AVRs are performed as minimally invasive operations to those who exclusively perform CAVR.

There are areas of concern for cardiothoracic surgeons regarding technique for $\mathrm{CPB}$ during MIAVR. These pertain to the limited cardiac exposure that has numerous theoretical risks (including placement of cannulae, adequate cardioplegia, and cardiac venting). Limited exposure might limit assessment of volume-loaded ventricles, deairing of the heart, placement of pacing wires, or a retrograde cardioplegia cannula. Transesophageal echocardiogram mitigates some of these problems, as well as instillation of $\mathrm{CO}_{2}$ into the operative field, anterior placement of pacing wires, and manipulation of empty ventricles on $\mathrm{CPB}$ to avoid right ventricular injury. The data do not allow us to comment on the conversion rate to full sternotomy for technical difficulties. Published data on conversions show it to be in the region of $1 \%$ to $3 \%{ }^{13}$ In the presence of similar CPB and crossclamp times in our cohort, it is likely that the surgeons undertaking the procedure have achieved the learning curve for the techniques.

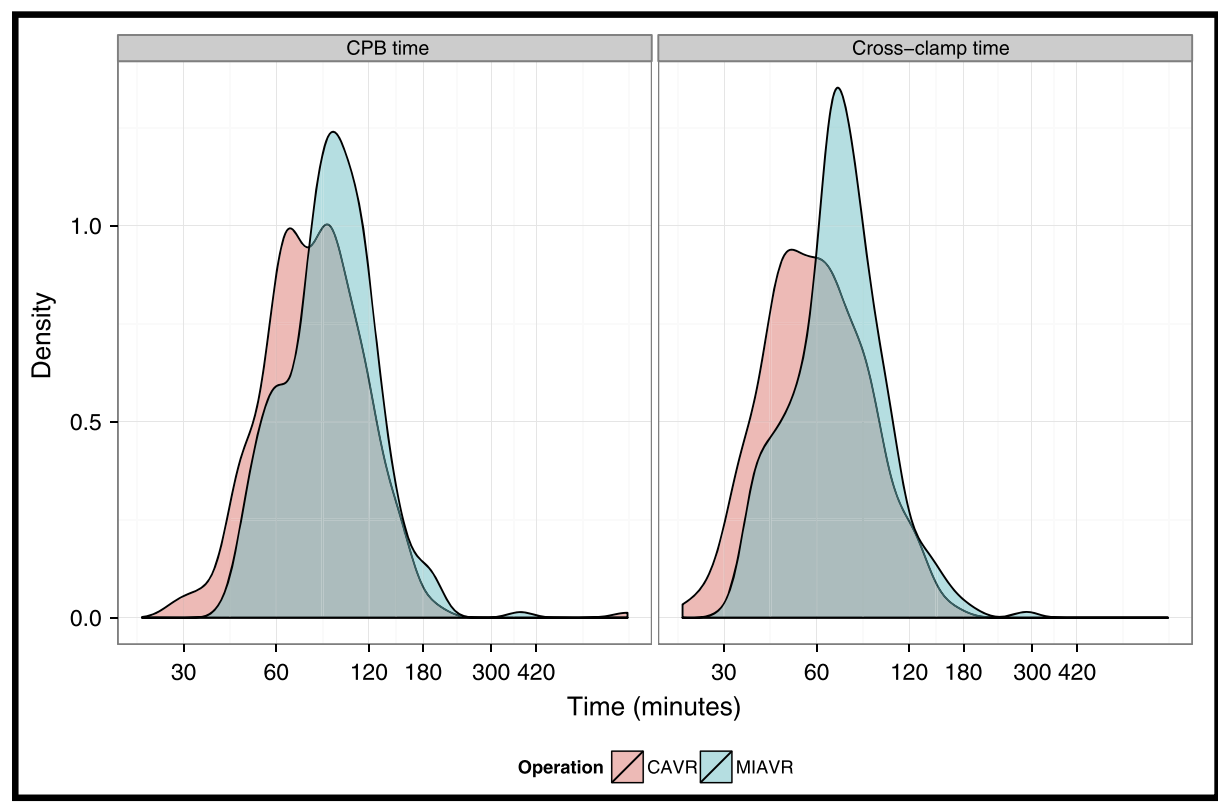

FIGURE 5. Kernel density distributions of cumulative CPB and cross-clamp times by AVR type in the propensity-matched cohort. Note the horizontal axis is on a logarithmic-transformed scale to aid interpretation. AVR, aortic valve replacement; CPB, cardiopulmonary bypass. 
There is apparent clinical equipoise between the two techniques. Minimally invasive AVR is safe, and multiple benefits have been reported in retrospective, propensitymatched, and randomized prospective studies. Previous studies of MIAVR have demonstrated reduced length of stay, with no change in mortality or operative times. ${ }^{12,27}$ The metaanalyses of multiple randomized trials have demonstrated decreased length of ITU stay and hospital stay, ${ }^{11,12}$ ventilation times, ${ }^{10}$ blood loss, ${ }^{10,11}$ and incidence of arrhythmias ${ }^{10}$ as well as improvement in pulmonary spirometry, sternal stability, and wound pain. ${ }^{15,28}$ However, a need remains for large adequately powered study to conclusively determine the advantages of MIAVR and in which patient population these benefits apply to.

Intuitively, these advantages might specially apply to higher-risk elderly patients with poorer physiological reserves and who might tolerate minimally invasive procedure better with a quicker recovery. ${ }^{29}$ The reasons for these reported benefits could be the maintenance of the bony continuity of the ribcage with preservation of respiratory dynamics, improved sternal stability, reduced pain, and less risk of chest and sternal infections. There might also be a reduction in bleeding as less bone marrow and periosteum are exposed with reduced cardiac dissection during surgical access.

The role of minimally invasive techniques in surgical AVR within the setting of sutureless valve technology and TAVI needs to be defined. Surgical AVR remains the criterion standard for patients requiring AVR. At present, although TAVI is only recommended for patients who are high-risk for surgical AVR, data from German registries have begun to demonstrate that "risk creep" is already occurring in transcatheter aortic valve procedures with some centers advocating expanding TAVI to younger lower-risk groups. ${ }^{30}$ In addition, a number of TAVI studies on intermediate-risk patients are on the way. The PARTNER II trial seeks to evaluate outcomes in patients with aortic stenosis and The Society of Thoracic Surgeons (STS) score of $4 \%$ to $6 \%$ and compare patients to CAVR. Patients with concomitant coronary artery disease will be randomized to percutaneous coronary intervention plus TAVI versus coronary artery bypass graft surgery plus AVR. As a noninferiority study, it will directly compare CAVR with transaortic (TAo) TAVI, transapical (TA) TAVI, or transfemoral-TAVI. Additional nested registries will also analyze outcomes in patients who are unsuitable for femoral access that are randomized to TA-TAVI or transaortic TAVI versus surgery. This strategy will seek to marry the advantages of the miniAVR access approach with balloon deployed TAVI device. Similarly, the Surgical Replacement and Transcatheter Aortic Valve Implantation (SURTAVI) trial will include patients older than 70 years with an STS score of $3 \%$ to $8 \%$.

The stent valve evolution will deliver a greater range of sizes of prosthesis through smaller totally percutaneous delivery systems. Novel valve designs are currently in early human studies to improve the incidence of paravalvular leak, for instance, with the addition of sealing cuffs to variations of nitinol frames. ${ }^{31}$ Numerous cerebral embolic protection devices aim to catch debris from the aorta and reduce the neurological morbidity from the procedure. ${ }^{32}$ Percutaneous closure devices for femoral access vessels are being routinely used with smaller delivery sheaths, and percutaneous left ventricular apex closure is in animal testing stages. ${ }^{33}$

If surgeons are to remain actively engaged in the heart valve team driving technical improvements in TAo and TA techniques, this is likely to begin with assessing the impact of MIAVR for surgical cases and likely to evolve into completely minimally invasive/port access implantation of transcatheter devices for high/intermediate-risk patients. One of the centers involved in this study has an active TAo-TAVI program, and it is likely that these techniques will develop in parallel. ${ }^{34}$ The presence of the heart team at units undertaking transcatheter valve procedures is increasing referrals of elderly comorbid patients now undergoing open AVR. . $35-37^{-37}$ However, a recent decline in CAVR work has been observed with up to $40 \%$ of aortic valve operations being performed by TAVI in 2012. ${ }^{37,38}$

Other authors have commented on the fact that although TAVI is an attractive and viable treatment for selected highrisk patients (eg, porcelain aorta, truly frail patients, previous cardiac surgery, patient grafts), surgical minimally invasive access AVR should be regarded as a criterion standard for all the rest of the patients. The functional outcomes are excellent, including valve durability, low incidence of stroke, and no paravalvular leak, which are of paramount importance. For octogenarians, the results of MIAVR are better than often assumed. ${ }^{29,38}$ Even when MIAVR is performed in patients with mean logistic EuroSCOREs of $17 \%$ (leading into the accepted TAVI range), the midterm survival is good, with inhospital mortality of $7.8 \%$ and freedom from all-cause death at 5 years at $72.4 \%{ }^{2,39}$ Outcomes in 175 octogenarians from a cohort of more than 1000 MIAVR patients demonstrated an operative mortality of $2 \% .{ }^{13}$

Minimally invasive AVR might offer additional advantages to the sutureless valve technologies that are perceived to be an alternative treatment for high surgical risk patients with severe aortic stenosis. Sutureless valves aim to minimize CPB and cross-clamp times by using novel rapid valve deployment techniques. One-year outcomes for the Edwards INTUITY Valve System as part of the TRITON trial report MIAVR in $48.8 \%$ of cases with overall cumulative survival of $92.5 \%$ at 9.8 months. The CPB and cross-clamp times are reported as 75 minutes and 46.6 minutes with $54 \%$ of cases being isolated AVRs. ${ }^{2,5}$ There are other INTUITY studies that are planned; with CADENCE-MIS, the benefits of MIAVR coupled with rapid deployment valves will be studied versus CAVR and standard valves. The use of Perceval S and ATS $3 f$ Enable has demonstrated similar reduction in bypass times of less than 30 minutes. ${ }^{3,40}$ Comparison with STS data show $60 \%$ decrease in operative time, which might reduce the effects on myocardial ischemia and hypoxia. ${ }^{27}$

Treatment of aortic valve disease is undergoing exciting and rapidly expanding technological developments that offer clinical and quality-of-life improvements to patients. A heart valve multidisciplinary patient-centered approach is vital to deliver best care to our patients.

\section{Future Research}

There seems to be genuine clinical equipoise between MIAVR and CAVR. Before widespread adoption of MIAVR 
can be recommended, there is a need for a well-constructed, adequately powered prospective randomized controlled study comparing MIAVR with CAVR. To demonstrate differences in mortality, the study would need to be powered to include very large number of patients. Pragmatic approach would involve high-risk patients in whom the clinical benefits would be more pronounced for the minimally invasive techniques. The benefits in terms of reduced ITU and hospital stay need to be balanced against the costs of mini techniques. Although majority of the procedure is performed using standard AVR equipment and valves, trials are already underway to compare the impact of sutureless valves. It is likely that the most benefit from these procedures will be in higher-risk elderly patients with limited physiological reserves. Increased patient preference for minimally invasive techniques and improvement in quality-of-life surveys are also important end points that will need to be considered in the randomized trials.

\section{CONCLUSIONS}

This propensity-matched study of minimally invasive aortic valve surgery is based on multicenter, "real-world" experience and shows that MIAVR is a safe and feasible procedure with excellent early and midterm outcomes for patients with aortic valve disease. In line with other authors, we have shown benefits in shorter hospital stay that might lead to more widespread use.

\section{REFERENCES}

1. Smith CR, Leon MB, Mack MJ, et al. Transcatheter versus surgical aorticvalve replacement in high-risk patients. $N$ Engl J Med. 2011;364: 2187-2198.

2. Kocher AA, Laufer G, Haverich A, et al. One-year outcomes of the Surgical Treatment of Aortic Stenosis With a Next Generation Surgical Aortic Valve (TRITON) trial: a prospective multicenter study of rapid-deployment aortic valve replacement with the EDWARDS INTUITY Valve System. $J$ Thorac Cardiovasc Surg. 2013;145:110-116.

3. Martens S, Sadowski J, Eckstein FS, et al. Clinical experience with the ATS 3 f Enable ${ }^{\circledR}$ Sutureless Bioprosthesis. Eur J Cardiothorac Surg. 2011; 40:749-755.

4. Kodali SK, Williams MR, Smith CR, et al. Two-year outcomes after transcatheter or surgical aortic-valve replacement. N Engl J Med. 2012 366:1686-1695.

5. Santarpino G, Pfeiffer S, Schmidt J, Concistrè G, Fischlein T. Sutureless aortic valve replacement: first-year single-center experience. Ann Thorac Surg. 2012;94:504-509.

6. Santarpino G, Pfeiffer S, Jessl J, et al. Sutureless replacement versus transcatheter valve implantation in aortic valve stenosis: a propensitymatched analysis of 2 strategies in high-risk patients. $J$ Thorac Cardiovasc Surg. 2014;147:561-567.

7. Aris A. Reversed "C" ministernotomy for aortic valve replacement. Ann Thorac Surg. 1999;67:1806-1807.

8. Svensson LG. Minimal-Access “J” or " $\mathrm{j}$ ” sternotomy for valvular, aortic, and coronary operations or reoperations. Ann Thorac Surg. 1997;64 1501-1503.

9. von Segesser LK, Westaby S, Pomar J, Loisance D, Groscurth P, Turina M. Less invasive aortic valve surgery: rationale and technique. Eur JCardiothorac Surg. 1999;15:781-785.

10. Murtuza B, Pepper JR, Stanbridge RD, et al. Minimal access aortic valve replacement: is it worth it? Ann Thorac Surg. 2008;85:1121-1131.

11. Brown ML, McKellar SH, Sundt TM, Schaff HV. Ministernotomy versus conventional sternotomy for aortic valve replacement: a systematic review and meta-analysis. J Thorac Cardiovasc Surg. 2009;137: 670.e5-679.e5.
12. Khoshbin E, Prayaga S, Kinsella J, Sutherland FW. Mini-sternotomy for aortic valve replacement reduces the length of stay in the cardiac intensive care unit: meta-analysis of randomised controlled trials. BMJ Open. 2011 Nov 24;1:e000266.

13. Mihaljevic T, Cohn LH, Unic D, Aranki SF, Couper GS, Byrne JG. One thousand minimally invasive valve operations: early and late results. Ann Surg. 2004;240:529-534.

14. Masiello P, Coscioni E, Panza A, Triumbari F, Preziosi G, Di Benedetto G. Surgical results of aortic valve replacement via partial upper sternotomy: comparison with median sternotomy. Cardiovasc Surg. 2002; 10:333-338.

15. Bonacchi M, Prifti E, Giunti G, Frati G, Sani G. Does ministernotomy improve postoperative outcome in aortic valve operation? A prospective randomized study. Ann Thorac Surg. 2002;73:460-465.

16. Kempfert J, Mohr FW, Walther T. Minimally invasive aortic valve surgery: where now and where to next? Expert Rev Cardiovasc Ther. 2009: 7:451-453.

17. Modi P, Hassan A, Chitwood WR Jr. Minimally invasive mitral valve surgery: a systematic review and meta-analysis. Eur J Cardiothorac Surg. 2008;34:943-952.

18. Hickey GL, Grant SW, Cosgriff R, et al. Clinical registries: governance, management, analysis and applications. Eur J Cardiothorac Surg. 2013; 44:605-614.

19. Roques F, Nashef SA, Michel P, et al. Risk factors and outcome in European cardiac surgery: analysis of the EuroSCORE multinational database of 19030 patients. Eur J Cardiothorac Surg. 1999;15: 816-822.

20. Moons KG, Donders RA, Stijnen T, Harrell FE Jr. Using the outcome for imputation of missing predictor values was preferred. J Clin Epidemiol. 2006;59:1092-1101.

21. van Buuren S, Groothuis K. MICE: multivariate imputation by chained equations in R. J Stat Softw. 2011;45:1-67.

22. Ho DE, Imai K, King G, et al. MatchIt: nonparametric preprocessing for parametric causal inference. Journal of Statistical Software. 2011;42:1-28.

23. Cohen J. Statistical Power Analysis for the Behavioral Sciences (Rev. Ed.). Hillsdale, NJ: Lawrence Erlbaum Associates, Inc; 1977:xv, 474.

24. Austin PC. A critical appraisal of propensity-score matching in the medical literature between 1996 and 2003. Stat Med. 2008;27:2037-2049.

25. Stuart EA. Developing practical recommendations for the use of propensity scores: discussion of 'A critical appraisal of propensity score matching in the medical literature between 1996 and 2003' by Peter Austin, Statistics in Medicine. Stat Med. 2008;27:2062-2065.

26. R Development Core Team. R: A Language and Environment for Statistical Computing. Vienna, Austria: the R Foundation for Statistical Computing; 2011:ISBN: 3- 900051-07-0. Available at: http://www.R-project.org/.

27. Tabata M, Umakanthan R, Cohn LH, et al. Early and late outcomes of 1000 minimally invasive aortic valve operations. Eur J Cardiothorac Surg. 2008;33:537-541.

28. Liu J, Sidiropoulos A, Konertz W. Minimally invasive aortic valve replacement (AVR) compared to standard AVR. Eur J Cardiothorac Surg. 1999;16(suppl 2):S80-S83.

29. Schmitto JD, Mohr FW, Cohn LH. Minimally invasive aortic valve replacement: how does this perform in high-risk patients? Curr Opin Cardiol. 2011;26:118-122.

30. Bleiziffer S, Mazzitelli D, Opitz A, et al. Beyond the short-term: clinical outcome and valve performance 2 years after transcatheter aortic valve implantation in 227 patients. J Thorac Cardiovasc Surg. 2012;143: 310-317.

31. Binder RK, Rodés-Cabau J. Transcatheter aortic valve replacement with the SAPIEN 3A new balloon-expandable transcatheter heart valve. JACC Cardiovasc Interv. 2013;6:293-300.

32. Carpenter JP, Carpenter JT, Tellez A, Webb JG, Yi GH, Granada JF. A percutaneous aortic device for cerebral embolic protection during cardiovascular intervention. J Vasc Surg. 2011;54:174.e1-181.e1.

33. Tang GH, Lansman SL, Cohen M, et al. Transcatheter aortic valve replacement: current developments, ongoing issues, future outlook. Cardiol Rev. 2013;21:55-76. 
34. Bapat V, Khawaja MZ, Attia R, et al. Transaortic transcatheter aortic valve implantation using Edwards Sapien valve: a novel approach. Catheter Cardiovasc Interv. 2012;79:733-740.

35. Grant SW, Devbhandari MP, Grayson AD, et al. What is the impact of providing a transcatheter aortic valve implantation service on conventional aortic valve surgical activity: patient risk factors and outcomes in the first 2 years. Heart. 2010;96:1633-1637.

36. Mack MJ. Transcatheter aortic valve implantation: changing patient populations and novel indications. Heart. 2012;98(suppl 4):iv73-iv79.

37. Subramanian S, Rastan AJ, Holzhey D, et al. Conventional aortic valve replacement in transcatheter aortic valve implantation candidates: a 5-year experience. Ann Thorac Surg. 2012;94:726-730.
38. Vasques F, Lucenteforte E, Paone R, Mugelli A, Biancari F. Outcome of patients aged $\geq 80$ years undergoing combined aortic valve replacement and coronary artery bypass grafting: a systematic review and meta-analysis of 40 studies. Am Heart J. 2012;164:410.e1-418.e1.

39. Dimarakis I, Rehman SM, Grant SW, et al. Conventional aortic valve replacement for high-risk aortic stenosis patients not suitable for trans-catheter aortic valve implantation: feasibility and outcome. Eur J Cardiothorac Surg. 2011:40:743-748.

40. Zannis K, Folliguet T, Laborde F. New sutureless aortic valve prosthesis: another tool in less invasive aortic valve replacement. Curr Opin Cardiol. 2012;27:125-129.

\section{CLINICAL PERSPECTIVE}

This study evaluated the outcomes of isolated minimally invasive aortic valve replacement (MIAVR) compared with conventional aortic valve replacement (CAVR) by performing a propensity-matched study from the UK National Database. Data were analyzed from seven volunteer centers from 2006 to 2012. Three hundred seven consecutive MIAVR patients were matched in a 1:1 ratio with CAVR patients. There was no statistically significant difference in the in-hospital mortality or 1-year survival. Although there was a significantly longer cross-clamp time in the MIAVR group, median postoperative length of stay was lower in the MIAVR patients by 1 day. The authors concluded that MIAVR was a safe alternative to CAVR with a shorter postoperative length of stay.

This is an excellent multicenter study evaluating MIAVR. The propensity matching is a strength of this article but does not fully compensate for selection bias. The authors recommended that a prospective, randomized study is needed to better evaluate MIAVR. Although this is true, unfortunately, it will be difficult for surgeons experienced in the minimally invasive technique and for patients to be randomized between MIAVR and CAVR. 